\title{
Una educación transformadora para El Salvador: propuesta y obstáculos para alcanzarla
}

\author{
Milton Ascencio Velásquez ${ }^{1}$, Universidad Don Bosco, El Salvador \\ Recibido: 3 de agosto, 2015. \\ Aprobado: 19 octubre, 2015.
}

\section{Resumen}

Este artículo tiene dos propósitos. Primero, exponer un punto de vista sobre el concepto educación de calidad en relación con la finalidad de la educación salvadoreña expresada en la Constitución Política de El Salvador. Se propone que una educación de calidad sea entendida como aquella que procura la formación de ciudadanos críticos y activos a partir del desarrollo del pensamiento crítico, con lo cual se logre dar respuestas a los problemas sociales actuales. El segundo propósito es argumentar que en el presente existen serios obstáculos para que el Estado salvadoreño garantice una educación transformadora a su población tales como acceso limitado al sistema educativo, alta deserción escolar, preparación docente limitada, así como la falta de consenso de país en torno a la educación, poca inversión en la educación e ideas reduccionistas que orientan los programas educativos gubernamentales únicamente a la competitividad laboral y la actualización tecnológica.

\section{Abstract \\ TRANSFORMING EDUCATION FOR EL SALVADOR: PROPOSALS AND OBSTACLES TO ACCOMPLISH IT}

This paper has two purposes. First, to present a point of view about the concept of quality education in relation to the goals of Salvadoran education as written in the Political Constitution of El Salvador. It is assumed that quality education is understood as that which procures the teaching of critical and active citizens using the development of critical thinking as a point of departure, which allows them to provide solutions to the current social problems. The second purpose is to argue that in the present there are serious obstacles for the Salvadoran State to guarantee a transforming education of its population such as the limited access to the educational system, a high school drop-out rate, limited training of the educators, as well as the lack of a nation-wide consensus regarding education, little investment in education and reductionist ideas which orient the government's educational programs towards labor competitiveness and technological updating only.

1 Milton Ascencio es máster en Lingüística del Inglés, Licenciado en idiomas (opción Enseñanza) y egresado del Doctorado en Educación de la Universidad de Costa Rica. Se desempeña como Director de la Escuela de Idiomas y como profesor de Lingüística Inglesa en la Universidad Don Bosco, de El Salvador. Contacto: milton.ascencio@udb. edu.sv.
Milton Ascencio Velásquez. Una educación transformadora para El Salvador: propuesta y obstáculos para alcanzarla. Revista Comunicación. Año 36, vol. 24, núm. 2, julio-diciembre, 2015. Tecnológico de Costa Rica. ISSN impreso: 0379-3974 / e-ISNN: 1659-3820.
PALABRAS CLAVE:

educación transformadora, calidad de la educación, educación ciudadana, pensamiento crítico, derecho a la educación, políticas educativas.

\section{KEY WORDS:}

transforming education, quality education, citizens' education, critical thinking, right to education, educational policies 
"El Salvador no ha conseguido construir una escuela pública de calidad que proporcione las bases para que las personas sean agentes de su propio desarrollo" (PNUD, 2013a, p. 138). De esta manera comienza el diagnóstico del PNUD sobre la educación salvadoreña, la cual exhibe cuatro aspectos débiles: en primer lugar, la educación en El Salvador continúa siendo desigual para todos, certificando la pobreza pero otorgando credenciales adecuadas a los no pobres. En segundo lugar, hay una formación inicial deficiente y capacitación limitada a lo largo de la carrera docente, la cual representa un obstáculo para que el sistema educativo pueda dar un salto cualitativo. En tercer lugar, la poca capacidad que el sistema educativo tiene para reducir los altos niveles de deserción, y en cuarto lugar pero no por eso menos crucial, la imposibilidad para trascender del ámbito simbólico en el discurso, al ámbito de la intervención concreta, de manera que incida en las personas en cuanto a sus deberes, responsabilidades, y sobre todo en su rol como ciudadanos en el proceso de transformación y solución a los problemas sociales.

Este último asunto que se señala, se refiere a la situación de violencia e inseguridad en la que El Salvador actualmente está sumergido, y que se ha agravado en los últimos años. En estos momentos, El Salvador es una de las naciones más violentas de la región latinoamericana. Su tasa de homicidios se estima en 70 muertes por cada 100 mil habitantes (PNUD, 2013b), el número de muertes violentas aumentó de 2,543 en el 2012 a 3,912 en el 2014 (BBC Mundo, 2014; "El Salvador cerró", 2015). Lo grave es que esta tendencia ha continuado al alza, con cifras sin precedentes en la historia reciente de El Salvador. De hecho, El Diario de Hoy (9 de septiembre) estima que para el 2015, la cifra podría llegar a 5,000 muertes violentas. A ello hay que agregar las extorsiones y amenazas de muerte ocurridas a casi el $80 \%$ de las micro y pequeñas empresas, lo cual ha ocasionado el cierre de negocios y la huida del país (PNUD, 2013a; "Más de 80 homicidios", 2015).

Esta situación suele atribuirse al accionar de miembros de pandillas o maras, debido a que operan con bastante impunidad y tienen estructuras conformadas por alrededor de 80,000 miembros, cuya edad promedio está entre los 15 y 16 años (PNUD, 2013a). Para el PNUD (2013b), el problema de las pandillas está relacionado directamente con la falta de oportunidades de educación, el desempleo y la migración al extranjero y posterior deportación al país. Muchos jóvenes ven las pandillas como referente de éxito y una forma de alcanzar prestigio social. Por eso, ámbitos como la familia y la escuela están ahora seriamente amenazadas por este problema ¿Cómo se puede, cambiar una situación de este tipo, mediante la educación?

Ahora bien, no sería justo pensar que la educación tiene toda la responsabilidad de erradicar la violencia e inseguridad social, pero es cierto que en muchos discursos se mantiene como fuente de convivencia y medio para que las personas se vuelvan agentes de transformación social.

Sin embargo, como lo señala el diagnóstico del PNUD (2013a, 2013b), la educación en El Salvador no ha conseguido ser ese medio de calidad para solucionar los problemas sociales. Y esto ocurre, básicamente, porque existen al menos tres razones que no le han permitido responder de forma adecuada a las condiciones actuales de vida de la población: a) hasta la fecha, no ha sido posible garantizar el pleno derecho a una educación transformadora, ya que todavía prevalece un acceso limitado al sistema educativo, alta deserción escolar y falta de preparación docente. b) la finalidad de los planes educativos recientes no ha sido coherente con el ciudadano que la Constitución Política de El Salvador demanda. c) La falta de acuerdos sobre una visión de educación para el país, la poca inversión en educación así como la idea reduccionista de que las políticas educativas deben ser orientadas únicamente hacia la competitividad o la actualización tecnológica, las cuales representan serios obstáculos para consolidar una educación transformadora como se entiende en este artículo.

Si se quiere una educación para resolver los problemas sociales, entre ellos la violencia, primero se debe revalorar el propósito de la educación salvadoreña, y luego, idear los medios para alcanzarlo, pues no sería posible obtener resultados diferentes mientras se continúe pensando y haciendo lo que hasta ahora no ha funcionado. 
La idea central en este trabajo es argumentar que la educación salvadoreña permitirá a sus ciudadanos volverse agentes de transformación social y dar solución a los problemas sociales, cuando las políticas y acciones educativas garanticen el acceso a una enseñanza transformadora la cual, mediante el desarrollo del pensamiento crítico, procure la formación de ciudadanos con capacidad de hacer frente a su realidad y orientar sus decisiones y acciones para favorecer la convivencia, así como para rechazar las diversas formas de violencia actuales.

Este artículo se ha estructurado en seis secciones. En la primera sección se aborda la finalidad de la educación, según se establece en la Constitución Política de El Salvador. La segunda sección expone cómo entender la capacidad del pensamiento crítico, sus alcances y enfoque para su desarrollo. En la tercera, se revisan los discursos de organismos e instituciones educativos sobre la capacidad crítica y formación ciudadana. En la cuarta, se discute qué entender por calidad de la educación, así como los avances en garantizar el derecho a recibirla. En la quinta sección se cuestiona si los programas educativos en El Salvador procuran una educación que garantice la formación del ciudadano que se demanda en la Constitución Política y, finalmente, en la sexta sección se exponen tres causas por las cuales El Salvador no posee las condiciones para lograr una educación transformadora. Se concluye con algunas ideas relacionadas con el fin de la educación salvadoreña y el pensamiento crítico, como un componente esencial para lograr una educación orientada a la transformación social.

\section{FINALIDAD DE LA EDUCACIÓN SALVADOREÑA}

La Constitución Política de El Salvador le indica al Estado salvadoreño que la educación en el país tiene seis fines acordes con la Declaración Universal de los Derechos Humanos. El artículo 55 de la Constitución declara que la educación en El Salvador debe: a) procurar el desarrollo integral de las personas; b) construir una sociedad democrática, justa y humana; c) inculcar los derechos humanos; d) combatir la intolerancia y el odio; e) dar a conocer la realidad nacional (Corte Suprema de Justicia, 1983). Además, el artículo 56 establece que todas las personas tienen el deber y el derecho de recibir un tipo de educación que garantice la formación de ciudadanos útiles. Sin embargo, dado que no es explícito el concepto de "ciudadano útil", seguidamente se hará una interpretación al respecto, antes de abordar el deber y el derecho a la educación.

Una revisión bibliográfica sobre los propósitos de la educación permite identificar al menos dos posiciones al respecto. Por un lado, para algunos autores, el propósito de la educación y de los centros educativos se orienta a la formación de capacidades y actitudes que preparen a los estudiantes para insertarse en el mundo laboral (Ferraz, 2012; McCowan, 2012; Rodríguez, 2006; Sacristán y Pérez, 2003; Steur, Jansen y Hofman, 2012). Sin embargo, se debe apuntar que la formación laboral no forma parte de los fines de la educación expresados en el Art. 55 de la Constitución Política de este país.

Por otro lado, está la postura de quienes proponen una pedagogía crítica, la cual coincide con los citados fines de la educación al expresar que los estudiantes deben ser preparados para convertirse en ciudadanos críticos y activos. Mientras que un ciudadano crítico es una persona capaz de analizar y cuestionar el orden social existente, un ciudadano activo orienta sus decisiones y actuación para favorecer la convivencia, la diversidad así como para rechazar las diversas expresiones de violencia y exclusión que caracterizan a la sociedad actual (Delgado, 2010; Ferraz, 2012; Giroux, 1990; Giroux y McLaren, 1998; Gutiérrez, 1985; Haydon, 2003; Johnson y Morris, 2010; McCowan, 2012; MacLaren y Giroux, 2003 y 2003b; Nussbaum, 2006; Perafán, 2014; Tenti, 2009).

La segunda postura es preferible para interpretar el ciudadano útil que la Constitución Política de El Salvador demanda, porque dicha posición no solo concuerda con los fines de la educación salvadoreña, sino que además presenta la educación como alternativa para la formación de ciudadanos que contribuyan a solucionar los problemas de la sociedad salvadoreña. Por tanto, para lograr que los salvadoreños se vuelvan ciudadanos críticos y activos, las acciones educativas han de estar orientadas a la formación de un pueblo con capacidad crítica, cuyas decisiones y hechos estén orientados por la justicia social, e impulsados hacia el bien común y la transformación 
social. En otras palabras, si se quiere formar ciudadanos críticos y activos, el desarrollo del pensamiento crítico es una condición determinante para lograr ese propósito.

\section{EL PENSAMIENTO CRÍTICO}

Según Bean (2011), el pensamiento crítico es un procedimiento reflexivo, constituido por dos procesos genéricos subyacentes: identificar y desafiar supuestos, así como explorar formas alternativas para pensar y actuar. Además, de acuerdo con Facione (1990), esta reflexión se forma de dos dimensiones: habilidades cognitivas y disposiciones afectivas, y se caracteriza por la indagación, el cuestionamiento de la evidencia, el sometimiento a la comprobación y la propuesta de solución ante problemas o situaciones inciertas. Según este autor, por un lado se encuentran las habilidades cognitivas centrales (la interpretación, el análisis, la evaluación, la inferencia, la explicación y la autoregulación ${ }^{1}$ ) y por otro, las disposiciones afectivas, las cuales implican el involucramiento en juicios críticos y en situaciones y propósitos fundamentados en valores personales y cívicos. Las disposiciones afectivas caracterizan a la persona por ser inquisitiva, estar bien informada, tener autoconfianza, ser de mente abierta, ser flexible, saber escuchar la opinión de los demás, ser honesto con los prejuicios propios, manifestar prudencia al emitir juicios y mostrar flexibilidad para reconsiderar puntos de vista propios. Esto sugiere que para desarrollar el pensamiento crítico no basta con crear oportunidades para ejercitar los procesos mentales, sino también crear las condiciones afectivas que faciliten esos procesos (Olivares, Saiz y Rivas, 2013).

Aunque desde la postura oficial (como se le denomina en Lim, 2011 y en Doughty, 2007) el pensamiento crítico se entiende como el análisis de argumentos junto con el razonamiento lógico sobre textos escritos y se ha desarrollado bajo un enfoque racional-instrumental, varios autores coinciden en la necesidad de potenciar el pensamiento crítico desde la educación bajo el supuesto que las habilidades asociadas a este proceso reflexivo son transferibles al campo profesional y que, además, motivan a la acción ciudadana (Boghossian, 2012; Crenshaw, Halke y Harper, 2011; Dwyer, Hogan y Stewart, 2012; Facione, 1990 y 2014; Elder y Paul, 2013; Levine,
2010; Lim, 2011; Nussbaum, 2006 y Posada, 2013). En tal sentido, Levine (2010) argumenta que "teaching for critical thinking is an essential element of professional education -of educating professionalssince it promotes reasoned judgment...future professionals must develop the habit of making difficult decisions about means and ends under conditions... which require moment-to-moment decision-making action" ( p. 72). Más allá de la vida profesional, Facione (1990) sostiene que el pensamiento crítico "is a liberating force in education and a powerful resource in one's personal in civic life...educating good critical thinkers...combines developing CT skills with nurturing those dispositions which consistently yield useful insights...the basis of a rational and democratic society" (p. 2). En términos similares, pero con mayor contundencia, Lim (2011) declara que "an education in critical thinking is the only education of which it can be truly said prepares the individual for democratic participation" (p. 784). Así las cosas, no parece que haya discrepancia en cuanto al potencial conferido a la educación para el desarrollo del pensamiento crítico y su importancia para la vida profesional y para la participación ciudadana.

En contraposición a un enfoque racional e instrumental, basado en modelos heurísticos uniformes y estándares preestablecidos, la capacidad del pensamiento crítico bajo el enfoque racional y práctico propicia la argumentación y la inclusión de diversas perspectivas. Se trata de ejecutar "a framework of reasoning principles that relates not just to what would be true for one to believe (theoretical reason), but also what would be morally desirable for one to do (practical reason)" (Lim, 2011 , p. 800). En palabras similares, es necesario moverse "from established criteria to the process of inquiry and communication that legitimates (at times) divergent alternative perspectives" (Bevan, 2009, p. 175), y así, enseñar a los estudiantes a participar en deliberaciones complejas como parte de experiencias significativas, dialógicas y comunicativas. La capacidad de pensar críticamente inicia con el cuestionamiento del propio sistema de valores para justificar tanto lo que se cree, como lo que se hace. Es más, se debe agregar que la capacidad crítica no se limita al mero cuestionamiento, sino que motiva y promueve la acción ciudadana a partir del consenso 
de sistemas de creencias alternativos sustentados en la razón. Steur, Jansen y Hoffman (2012) explican que no se trata de decidir entre lo bueno y lo malo, sino de educar a los estudiantes para que aprendan a cuestionar sus propias creencias y reconozcan que ellos "need to know from where their beliefs stem, and...to know that other people might hold different beliefs" (p. 866). De manera similar, Bevan recuerda que "success in inquiry is not based on a monist rational framework of truth, or pervasive right or wrong...but depends on an understanding of multiple perspectives that may be in conflict with one's dogma" (2009, p. 177).

Además, para asegurar que el desarrollo del pensamiento crítico sea efectivo para la formación de ciudadanos argumentativos, se necesitan el contexto y la experiencia significativa de cada uno. Con frecuencia se ignora el contexto de la problemática abordada, como sucede bajo un enfoque racionalinstrumental, "to the extent that students have no idea of what they are arguing about despite the handy framework" (Bevan, 2009, p. 175). Por ello, Lim (2011) insiste en que, para cultivar el pensamiento crítico realmente, se necesitan oportunidades para mirar el mundo desde perspectivas distintas y hasta en contradicciones, que demuestren y reflejen los intereses en competencia y realidades de los distintos grupos sociales. Si el objeto del pensamiento crítico es capacitar a las personas para comprender, analizar y resolver problemas que se afrontan todos los días en una sociedad democrática, una presentación de situaciones descontextualizadas y, en muchos casos, sin relación con los problemas sociales solo puede Ilevar a un tipo de racionalidad instrumental, moralmente indiferente y emocionalmente apático, lo cual resulta en la despolitización de la democracia (Lim, 2011). En esa misma línea, Giroux (1990) previene de enseñar de manera aislada y dispersa e invita a "capacitar a las personas para interpretar críticamente sus mundos personales y sociales y, de este modo, mejorar su habilidad hasta estar en condiciones de poner en entredicho los mitos y creencias que estructuran sus percepciones y experiencias" (p. 192).

En esa misma línea de pensamiento, Perafán (2014) propone que, para la formación de ciudadanos, los procesos educativos deben generar un tipo de experiencia significativa que apele a la emoción y pro- duzca un sentido de responsabilidad. Por un lado, se necesita de un tipo de experiencia que, mediante la imaginación y la creatividad, permita no solo adentrarse en la vida de los demás, sino también generar sensibilidad, empatía y una razón compasiva que resulte en solidaridad. Esta propuesta de Perafán pretende brindar situaciones que incomoden a quien las experimenta, con el objetivo de engendrar la obligación moral para actuar. Por otro lado, el sentido de responsabilidad implica que los estudiantes deben tener oportunidades para expresarse y ser reconocidos mediante situaciones orientadas a la búsqueda de posibles alternativas, frente a diferentes problemas de su comunidad, ya sea de forma individual o colectiva. En acuerdo con Perafán (2014), para que el pensamiento crítico sea un medio eficaz para formar ciudadanos críticos y activos, debe tener lugar a partir de una experiencia educativa que permita comprender los problemas de otros, ponerse en la situación de los demás y motivar a la acción ciudadana. Solo entonces se puede hablar de una educación transformadora, que otorgue relevancia al desarrollo del pensamiento crítico como medio para la formación de ciudadanos activos.

Por otro lado, las ideas sobre el pensamiento crítico expuestas permiten poner en perspectiva los discursos que emanan desde los organismos e instituciones educativas. Si se acepta la idea de que los estudiantes pueden transferir la capacidad del pensamiento crítico a su vida real, y que dicha capacidad es determinante para la formación de ciudadanos críticos y activos, entonces habrá que indagar cómo estos aspectos adquieren importancia en los discursos y acciones que las instituciones y agentes de la educación proponen.

\section{DISCURSOS: CIUDADANOS CRÍTICOS Y ACTIVOS}

Tanto la capacidad crítica como la formación de ciudadanos activos aparecen como elementos importantes en cada una de las declaraciones de propósitos educativos, ya sea desde los organismos internacionales como de las instituciones educativas salvadoreñas. En el ámbito internacional, tanto la UNESCO como el Sistema de Integración Centroamericana (SICA) se muestran a favor del desarrollo de la capacidad crítica y la formación de ciudanía 
activa. La UNESCO por su parte, señala la necesidad de enfocar la educación hacia la promoción de habilidades y actitudes para la participación ciudadana y política "a fin de que los estudiantes no solo aprendan contenidos, sino también aprendan a mejorar sus relaciones sociales y su involucramiento en la sociedad de la que son parte" (UNESCO, 2013, p. 27). Asimismo, la Política Educativa Centroamericana 2013-21 (PEC) hace referencia a una educación que busque "desarrollar la creatividad, el pensamiento indagatorio y crítico, y la capacidad para utilizar el conocimiento en la resolución de problemas...fomentar la formación humana y ciudadana...para la sana convivencia y para el ejercicio responsable de la libertad" (Sistema de la Integración Centroamericana, 2014).

En el ámbito salvadoreño, el marco legal así como el discurso oficial sientan posición sobre la capacidad crítica y la formación de ciudadanos activos. La Ley General de Educación de El Salvador establece que se deben "cultivar la imaginación creadora, los hábitos de pensar y planear...y el desarrollo de la capacidad crítica" (Asamblea Legislativa de El Salvador, s.f., Art. 3, literal d). También, el Ministerio de Educación de El Salvador expresa que el desarrollo curricular basado en competencias orienta a "que el alumno adquiera los aprendizajes significativos (saberes) por medio de la solución de problemas contextualizados y el desarrollo del pensamiento crítico" (MINED, 2008, p. 10). Específicamente, en el programa de los Estudios Sociales y Cívica se asocia la reflexión crítica con las condiciones socio-políticas, económicas y culturales, con el objetivo de crear conciencia y entendimiento del porqué y cómo de los hechos, "promoviendo en el alumnado situaciones morales y éticas frente al análisis de los problemas del país y de la región, para que rechace toda forma de falsedad y que adopte una posición comprometida [con] la democracia y la paz" (MINED, 2008, p. 27). Además, el Plan Social Educativo 2009-2014 de este país, propone un modelo "que propicie una conciencia crítica y reflexiva...Esta formación histórico-cultural será acompañada de una formación humanista que facilitará al educando la interiorización crítica y reflexiva de un marco ético, cuya práctica ocurrirá en los diferentes escenarios de la vida" (MINED, 2009, p. 37).
Las instituciones de educación superior en El Salvador también hacen eco del deseo de formar ciudadanos críticos y activos, aunque matizados con una variedad de discurso coincidente como lo demuestran los siguientes ejemplos. En el sector público por ejemplo, la Universidad de El Salvador declara ser una institución "transformadora de la educación superior y desempeñar un papel protagónico relevante, en la transformación de la conciencia crítica y propositiva de la sociedad salvadoreña" (Universidad de El Salvador, sitio web, s.f.). En el sector privado por su parte, la Universidad Don Bosco establece, en su Modelo Educativo, que se opta por una educación caracterizada por ser "crítica y propositiva orientada a la búsqueda de la verdad mediante la investigación y el diálogo...implica un acercamiento crítico a la realidad...una educación históricamente situada, que busca que sus actores se hagan cargo de la realidad" (Universidad Don Bosco, sitio web, s.f.). Por su parte, la Asociación de Universidades Privadas de El Salvador, AUPRIDES, expresa que los esfuerzos desde las instituciones que agrupa son orientados hacia la búsqueda de la verdad científica y la solución de problemas del país así como a la formación del profesional "como un actor solidario con el cambio social que requiere nuestra realidad" (AUPRIDES, sitio web, s.f.).

Al revisar cada una de estas declaraciones, no parece haber discusión en cuanto al deseo de formar personas que posean no solo capacidad crítica, sino que además sean ciudadanos comprometidos con los cambios que la sociedad salvadoreña necesita. Ahora bien, dada la importancia que se confiere al desarrollo de la capacidad crítica en la formación de ciudadanos, sería de esperar que las políticas y acciones educativas que hasta la fecha se han llevado a cabo, tengan como finalidad garantizar el derecho que todos los salvadoreños tienen no solo de acceder al sistema educativo, sino también de recibir un tipo de educación acorde con lo antes anotado por dichos organismos e instituciones. Por ello, en las siguientes secciones se revisa qué entender por derecho a la educación y en qué medida ese derecho ha sido garantizado a los salvadoreños. 


\section{EL DERECHO A UNA EDUCACIÓN TRANSFORMADORA}

De regreso al artículo 56 de la Constitución Política de El Salvador, ahí se establece que los ciudadanos no solo tienen el derecho a la educación, sino también el deber de recibirla. Se podría pensar que el deber al cual se hace referencia está determinado por la responsabilidad de cada individuo para recibirla, mas no en el sentido de imposición. En todo caso, sería el Estado salvadoreño quien tiene la obligación, por ley, de garantizar el acceso a la educación para sus ciudadanos como un derecho inalienable de todos los seres humanos. No obstante, en el discurso oficial se suele hablar de una educación obligatoria, como sucede en el artículo 5 de la Ley General de Educación. Sin embargo, Dubet (2005) cuestiona si es apropiado obligar a los estudiantes a asistir a la escuela cuando esta no puede garantizar un bien común a todos. Para Dubet "es necesario definir lo que la escuela obligatoria debe garantizar obligatoriamente a todos los alumnos [caso contrario] los alumnos tendrían toda la razón en rechazar las pruebas, pues es el bien común el que crea la obligación" (p. 58). Por lo anterior, el Estado salvadoreño está obligado no solo a garantizar el acceso a la educación de sus habitantes, sino también a brindar educación que produzca el sentido del deber y que se constituya un bien común para todos.

La idea de obligatoriedad puede hacer perder de vista que lo importante es el derecho a una educación, cuyo propósito sea el desarrollo integral de la persona y capacitarle para dar respuestas pertinentes a los problemas sociales, por encima de otros intereses. Con frecuencia el derecho a la educación se percibe solo en términos de cobertura o rendimiento académico, quizás para mejorar la imagen y las estadísticas de los gobiernos ante los organismos internacionales. No obstante, no basta con asegurar que cada vez sean más las cantidades de personas que ingresan y permanecen en el sistema educativo si el tipo de educación que se les brinda no contribuye a mejorar sus condiciones de vida de las personas ni tampoco a que los estudiantes se conviertan en ciudadanos críticos y activos. Tal situación solo haría que se continúen usando grandes cantidades de fondos públicos para entretener a los estudiantes, sin que ello se revierta en beneficio para la sociedad. En tal sentido, la UNESCO (2013) señala que "cada vez más los nuevos criterios con que se deben juzgar los avances en educación remiten a la calidad más que a la simple expansión de la educación" (p. 17). Según este organismo, una educación de calidad es parte constitutiva del derecho a la educación orientada por tres criterios: a) desarrollar las capacidades individuales, b) promover los valores humanos (igualdad, respeto a la diversidad, tolerancia, la no discriminación y el respeto al bien común) y c) formar personas que sean socialmente competentes.

No obstante, acá se propone replantear lo que se entiende por calidad de la educación. Dicho concepto debe ser entendido como una acción educativa transformadora cuyo objetivo primordial sea formar ciudadanos quienes, a partir del desarrollo de sus capacidades intelectuales, promuevan los valores humanos: el respeto a la diversidad, la convivencia, la tolerancia y la no discriminación. Por lo tanto, el interés debe ser puesto sobre un tipo de educación que responda a cómo se desarrollan las capacidades de los estudiantes, cuáles son esas capacidades, cómo se promueven los valores humanos y qué se hace para desarrollar ese ciudadano que la Constitución Política salvadoreña demanda. Esta idea implica que una educación transformadora, como se entiende aquí, está íntimamente ligada con el quehacer docente más que con el incremento del número de estudiantes que ingresan al sistema educativo. Sin embargo, aunque el derecho a la educación se quisiera determinar por acceso de las personas al sistema educativo, los datos que se presentarán en breve muestran que en El Salvador tal derecho no se garantiza a la población en su totalidad, pues no solo el acceso y permanencia en el sistema educativo aún son limitados, sino que persiste un alto porcentaje de salvadoreños que todavía son analfabetas. Además, una educación como acá se plantea no se puede garantizar si la preparación docente es aún deficiente y si los planes educativos gubernamentales no están orientados hacia la formación de ciudadanos críticos y activos como se expondrá más adelante.

Los datos siguientes dan evidencia de lo insuficientes que han sido los esfuerzos en El Salvador para ampliar la cobertura y reducir la deserción escolar, mejorar el rendimiento académico y reducir el analfabetismo. En primer lugar, interesa comparar la tasa 
de matrícula con la deserción en los diferentes niveles de la así llamada "educación obligatoria". En términos generales, el $10 \%$ de la población pobre únicamente ha logrado obtener tres años de escolaridad (de un total de once años) en comparación con diez años alcanzados por el $10 \%$ de la población más rica (PNUD, 2013b, p. 39). Además, "en el país hay un 28\% de jóvenes entre 16 y 24 años con privación educativa severa, es decir, que no han terminado siquiera la educación primaria" (PNUD, 2013b, p. 42). El PNUD señala también que, para el 2011 solo el $54.2 \%$ ingresó al nivel parvulario y según ERCA (2014), en el 2013 este porcentaje fue del $56.4 \%$.

Asimismo, el PNUD (2013b) señala que en el año 2012, la matrícula en primaria alcanzó el 93.7\% de la población requerida y para el 2013, bajó al 88.2\% (ERCA, 2014).

En educación secundaria, el porcentaje de matrícula para el 2013 fue solo del 63.3\%, el mayor desde el 2004.

Lo anterior demuestra que asegurar la permanencia escolar en El Salvador continúa siendo un problema. El PNUD indica que "El Salvador forma parte del grupo de países caracterizado por un abandono escolar temprano... la deserción se da antes de terminar la primaria, lo que profundiza los costos privados asociados a la deserción...como ingresos futuros perdidos por no completar los diferentes ciclos educativos" (2013a, p. 158). Las estadísticas muestran que el promedio de deserción en la primaria es $5.2 \%$ desde el 2004 hasta el 2013. En el nivel de secundaria es en el que se identifica una mayor tasa de deserción, con un promedio de 7.3\% del 2004 al 2013. Para este último año, la deserción subió al 7.9\%, es decir, un $0.6 \%$ más que el promedio.

Esos porcentajes tan bajos de matrícula y esos promedios tan altos de deserción, revelan cuán difícil resulta que la educación salvadoreña se convierta en la llave para abrir oportunidades de empleo, de movilidad social, de bienestar para los habitantes y que además, se vuelva un medio para la formación de ciudadanos capaces de dar solución a los problemas del país. De hecho, el PNUD (2013b) advierte que " 6 de cada 10 niños que ingresan al sistema educa- tivo llegará a noveno grado, y solo la mitad de ellos logrará terminar el bachillerato (p. 39).

En segundo lugar, el rendimiento académico de los estudiantes salvadoreños que egresan del sistema educativo también sigue siendo deficiente. Los resultados de la Prueba de Aprendizaje y Aptitudes para Egresados de Educación Media (PAES) dan cuenta del bajo rendimiento de los estudiantes que terminan la secundaria. Desde la implementación de la PAES, en 1997, el promedio de resultados apenas alcanza la nota de aprobación 5.0 a nivel nacional (en una escala del 1 al 10). En el 2014, el promedio nacional fue de 5.2 (5.3 en el 2013, 5.0 en 2012, 4.8 en 2011, 5.1 en 2010; MINED, 2009). Debe apuntarse que la educación privada (bilingüe y religiosa) es la que tiende a obtener mejores resultados en la PAES que la educación pública en cada una de las áreas evaluadas, aunque de igual manera se tiende a la reprobación (MINED, 2009). Aun así, se debe tener en cuenta que la educación privada no está al alcance de las grandes mayorías. Únicamente el $16 \%$ de la población salvadoreña, y especialmente en zonas urbanas, tienen la posibilidad de acceder a centros de educación privada (PNUD, 2013a). Otro ejemplo del bajo nivel de rendimiento académico es que El Salvador "se encuentra en la posición 33 entre 37 países en la Prueba de desempeño académico de Matemáticas y Ciencias (TIMSS)" (PNUD, 2013b, p. 39).

En tercer lugar, los datos sobre alfabetización de adultos salvadoreños tampoco invitan al triunfalismo. El Plan Social Educativo 2009-2014 tenía como meta reducir el analfabetismo al $10 \%$ en el 2010 y al 4\% en el 2015. Sin embargo, para el año 2012 la población en el área urbana de quince años en adelante, que sabe leer y escribir, no era mayor del $90.9 \%$ mientras que en el área rural no sobrepasó el 75.7\% (PNUD, 2013a, p. 101). Estos datos han variado muy poco en una década. Si bien para el 2004 el porcentaje total de población alfabetizada era del $83 \%$, para el 2013 era aún del 88\%. Estos sugiere que las políticas educativas en El Salvador no están enfocadas para erradicar el analfabetismo, ya que después de una década aún hay un $12 \%$ de la población sin saber leer y escribir, mientras que en otros países de la región como Belice, Costa Rica y Panamá el porcentaje de esta población no alfabetizada es menor 
al $2.5 \%$. Se debe reconocer que el analfabetismo en El Salvador se agravó con el período del conflicto armado (de 1980 a 1991), durante el cual el 68\% de población infantil no pudo ingresar al sistema educativo y la tasa de matrícula en secundaria no superó el $27 \%$ a nivel nacional. Ello ocasionó que en la zona rural, el $40 \%$ de la población no alcanzara ni un año de escolaridad, y que menos del 1\% completara la secundaria (PNUD, 2013a).

Como se mencionó anteriormente, una educación orientada a la transformación y la solución de los problemas sociales está asociada con la preparación docente; sin embargo, esta también es aún limitada. En El Salvador solo el 85\% de los profesores tiene una formación universitaria, comparado con otros países como Costa Rica (98\%) y Uruguay (100\%) (PNUD, 2013a, p. 146). Además, los datos presentados sobre la Evaluación de las Competencias Académicas y Pedagógicas (ECAP), cuya aprobación es requisito para egresar de todos los programas de profesorado en El Salvador, muestra no solo que el número de candidatos para ser docentes que se someten a esa prueba ha disminuido, sino que el promedio obtenido a lo largo de los años tiende a la reprobación (PNUD, 2013a). Esto es preocupante si, como lo señala la UNESCO, "el pilar fundamental de la calidad educativa son las capacidades profesionales docentes: si los alumnos no se encuentran en sus aulas con docentes capaces...no se producirá un genuino mejoramiento de la calidad educativa" (2013, p. 25). A lo anterior habrá que agregar otros agravantes como los bajos salarios, condiciones laborales deficientes, exceso de horas de trabajo, programas de formación obsoletos y falta de actualización profesional.

Las estadísticas presentadas dan cuenta, en parte, del porqué en El Salvador no están dadas las condiciones para alcanzar una educación transformadora. No es posible garantizar el derecho a la educación y menos a una educación transformadora mediante la cual se logren formar los ciudadanos críticos y activos anhelados, mientras el acceso a la educación, la permanencia, la alfabetización y la formación profesional docente aún representan serias dificultades. Esto reclama un replanteamiento no solo de la finalidad de la educación salvadoreña, sino también de sus actuales políticas educativas, que pretenden responder a las necesidades y los problemas sociales actuales. Sin embargo, como se verá a continuación, las políticas educativas salvadoreñas tampoco logran este cometido.

\section{PLANES Y ACCIONES EDUCATIVAS}

Las políticas educativas en El Salvador, con frecuencia denominadas reformas educativas, han sido en realidad acciones y programas que han emanado de visiones de gobierno más que de una visión de país. En 1940 se hizo la primera Reforma Educativa a partir de la cual se introdujeron planes de estudio, nuevos programas y una capacitación acelerada de profesores (MINED, 2009). Hacia finales de los años sesenta, una nueva reforma educativa reorientó la educación hacia la formación técnica. En 1968, bajo la dirección de Walter Béneke, se reestructuró la educación básica en tres ciclos (tres años cada ciclo), se implementaron los bachilleratos diversificados de tres años en áreas de trabajo como Hostelería y Turismo, Industrial, Contabilidad, Secretariado, etc. y se introdujo la Televisión Cultural Educativa como primer componente tecnológico en la educación salvadoreña (MINED, 2009). Con esta decisión se hizo perceptible el deseo de que la educación estuviera al servicio de la economía y de esa manera que el país se insertara en un modelo de industrialización que requería mano de obra calificada. Según el PNUD (2013b), esta reforma trajo consigo mejora de los indicadores de crecimiento en las zonas urbanas, no así en las áreas rurales, aunque su impacto positivo fue mermado a consecuencia de la guerra civil durante la década de los ochenta.

Pasado el conflicto armado de 1980-1990 y durante los siguientes veinte años se han producido intentos por enrumbar la educación en El Salvador. Durante la década de 1995-2005 se llevó a cabo el Plan Decenal de la Reforma Educativa como resultado de una consulta ciudadana dirigida por la Comisión de Educación, Ciencia y Desarrollo a lo largo del año 1995. Dicho plan estuvo acompañado de nuevas leyes y reglamentos tales como la Ley de Educación Superior en 1995, la Ley General de Educación y la Ley de la Carrera Docente, ambas en 1996 (MINED, 2009). Este Plan de Reforma Educativa 1995-2005 fue acompañado de otras acciones para ampliar la cobertura, mejorar la calidad educativa, modernizar la institución y formar en valores, las 
cuales significaron el preámbulo para el inicio de un nuevo plan de educación (MINED, 2005).

En 2005 inició el Plan Nacional de Educación 2021. La Sección III del documento expresa sus fundamentos y hace explícito su propósito: educar para ocupar posiciones elevadas en el índice de desarrollo humano en la escala mundial. Aún más, es una educación "para lograr un alto y sostenido crecimiento económico, el cual es base e instrumento para asegurar el desarrollo social y la estabilidad de nuestro sistema político" (MINED, 2005, p. 11). Con ese fin, se propusieron cuatro objetivos: a) desarrollo integral de la persona en lo físico, lo emocional, lo social, lo moral y lo espiritual; b) escolaridad de once grados para toda la población; c) formación técnica y tecnológica; d) desarrollo de la ciencia y la tecnología para el bienestar de la sociedad. Para lograr dichos objetivos, el plan agrupaba un conjunto de políticas educativas en cuatro líneas estratégicas de las cuales interesa la tercera, pues encierra de mejor manera el propósito principal de este plan educativo al enfatizar la competitividad a partir del desarrollo técnico y tecnológico.

En esta línea se establece que la competitividad tiene sus bases en competencias básicas entre las que menciona el razonamiento, el pensamiento crítico, la investigación y la resolución de problemas. Sin embargo, en ningún sitio queda claro como el razonamiento y el pensamiento crítico se desarrollarían. Contrario a ello, sí se explica que esta línea estratégica se orientaría al aprendizaje del inglés como una segunda lengua, el fortalecimiento de la red de soporte tecnológico, mejoramiento de la educación técnica y tecnológica así como la adaptación de la tecnología para aumentar la productividad. Esta línea estratégica encierra un componente ideológico determinante que orienta el diseño curricular al declarar que se pretende "la formación del capital humano en las áreas técnicas y tecnológicas,... [ya que] el uso de la ciencia y la tecnología para el desarrollo productivo del país, son factores esenciales para lograr una mayor competitividad" (MINED, 2005, p. 17).

Por un lado, considerado en su totalidad, el Plan Nacional de Educación 2021 no parece coincidir con los fines de la educación nacional expuestos en el artículo 55 de la Constitución Política de El Salva- dor. Los fines de la educación en dicho artículo de la Constitución Política hacen referencia al desarrollo integral de la persona, los derechos humanos, una sociedad justa y humana, etc. Sin embargo, aunque el Plan Nacional de Educación 2021 declara que los seis fines de la educación nacional fueron la "guía inequívoca" (MINED, 2005, p. 8) para su elaboración, el discurso que caracteriza a dicho plan resalta la competitividad y la producción más que alguno de los fines de la educación. Al considerar cada una de las políticas en el documento, ninguna de ellas aborda de forma particular y concisa alguno de los seis fines de la educación nacional declarados en la Constitución Política. De manera puntual, las políticas expresadas en este plan buscan la ampliación del acceso y la cobertura, la certificación del rendimiento académico, aumentar la competitividad y mejorar la gestión educativa.

Por otro lado, y en relación con el artículo 56, habría que indagar sobre la utilidad que se espera del ciudadano sujeto de una educación orientada por políticas de tal naturaleza. Si la utilidad ciudadana se deduce únicamente a partir de lo expresado, que la educación es el medio "para lograr un alto y sostenido crecimiento económico, el cual es base e instrumento para asegurar el desarrollo social y la estabilidad de nuestro sistema político" (MINED, 2008, p. $11)$, entonces se corre el riesgo de que la educación esté orientada solo hacia la formación de mano de obra calificada, la generación de trabajadores competitivos que funcionen de acuerdo con las exigencias del mercado y la unificación de pensamiento, quizá acrítico, que asegure la continuidad del orden social, económico y político establecido. En tal caso, no sería prioritario lograr una educación orientada a la formación de ciudadanos con capacidad de analizar el porqué de la situación social actual, de sus formas de pensar y actuar así como de sus posibles consecuencias. Probablemente tampoco sería una prioridad orientar la formación para el fomento del derecho a la expresión, la convivencia participativa o la formación de ciudadanos activos que orienten sus decisiones y acciones para promover la convivencia.

Con la llegada del primer gobierno de izquierda al poder, en el año 2009, el Plan Nacional de Educación 2021 pierde apoyo y aparece un nuevo proyecto: el Plan Social Educativo 2009-2014. Este plan 
propone un rediseño de la escuela y el aula lo cual permita no solo cambiar la forma en la que se enseña, sino también conectar con la comunidad, adquirir y reafirmar valores de respeto a la diversidad, a la identidad, "mediante el poder del conocimiento, de una instrucción que emancipa y libera" (MINED, 2009, p. 12). A partir de una pedagogía alternativa, este plan busca generar "espacios de reflexión entre los actores del proceso... en forma tal que el alumno sea el propio protagonista de su proceso formativo, orientado y guiado por el grupo docente,... con el apoyo de la familia y de la comunidad" (MINED, 2009, p. 14). Además, se propone invertir el orden de prioridades. Primero, si antes se hacía énfasis en el crecimiento económico, en el nuevo plan se pone a la cultura y la educación como condición prioritaria para toda transformación educativa, seguida por la participación, luego el plano político y finalmente el plano económico. Bajo esta idea, un proceso educativo reconoce "la cultura como base del desarrollo; a la familia y a la comunidad como sustento de organización social; y a la política y a la economía como medios para lograr lo anterior" (MINED, 2009, p. 28). Segundo, si en el plan anterior se buscaba formar un ciudadano competitivo, ahora se propone que "el sistema educativo formará ciudadanos con juicio crítico, capacidad reflexiva...que les permita transformar la realidad social y valorar y proteger el medio ambiente...una formación humanista que facilitará al educando la interiorización crítica y reflexiva de un marco ético" (MINED, 2009, p. 37).

Para sacar adelante el Plan Social 2009-2014, se proponen cinco fuerzas impulsoras y seis líneas estratégicas las cuales se traducen en siete programas insignia. Primero, las fuerzas impulsoras se enfocan en mayor inversión, administración eficiente, concertación social, participación de la sociedad y evaluación de resultados. Segundo, las líneas estratégicas que dan paso a los programas son: a) acceso y permanencia en el sistema educativo; b) diseño curricular pertinente; c) dignificación del magisterio; d) fortalecimiento de la gestión institucional; e) formación permanente para jóvenes y adultos; f) fortalecimiento de la educación superior; g) fortalecer la investigación, ciencia y tecnología. No obstante, el Plan Social Educativo 2009-2014 también debe ser valorado en cuanto a su correspondencia con la Constitución Política y la formación de los ciudadanos.

En primer lugar, no está claro como este proyecto pretende formar la capacidad crítica de los ciudadanos a partir de sus líneas estratégicas y los programas propuestos. Aunque una de sus líneas estratégicas propone diseñar un currículo pertinente, las acciones propuestas son generales, en el orden de revisar y actualizar el currículo existente, crear materiales didácticos, dar asesoría pedagógica, etc. sin aclarar la pertinencia a la cual se hace referencia y sin precisar cómo el currículo responderá al desarrollo de capacidades, la promoción de valores humanos o la formación ciudadana. Además, aunque en otra línea estratégica se propone la formación permanente de jóvenes y adultos, las acciones estratégicas propuestas se enfocan solo en la alfabetización y la preparación para el trabajo. Sin embargo, Ilama la atención que sea desde la línea estratégica de investigación, ciencia y tecnología que se pretenda mejorar la educación al dotar de computadoras portátiles a estudiantes y docentes. Según se declara, esta es "una acción particularmente importante, y que podría significar el necesario salto de calidad en nuestro sistema educativo, en cuanto al rediseño en el aula y la modernización pedagógica...que puede permitir mejorar las condiciones de aprendizaje" (MINED, 2009, p. 55).

No se podría estar en mayor desacuerdo con dicha proposición. Mejorar la educación no se puede garantizar por el hecho de equipar estudiantes y aulas con computadoras cuando aún existen carencias de infraestructura apropiada para ello y capacidad pedagógica para un uso pertinente de la tecnología. Supeditar el mejoramiento de la educación al uso de la tecnología podría ser engañoso, especialmente cuando se propone formar a los profesores en el uso de las computadoras, pero no se aclara cómo se usarán para lograr fortalecer los derechos humanos y la formación de ciudadanos. Si bien es cierto que las computadoras facilitan el acceso a más información, ello no se traduce necesariamente en el fortalecimiento de los derechos humanos y la formación de ciudadanos críticos y activos, sin mejorar la capacidad didáctica-pedagógica de los docentes. Antes de equipar con tecnologías a las escuelas se requiere capacidad didáctica y claridad pedagógica, 
que superen concepciones y prácticas tradicionales del actual sistema educativo.

En segundo lugar, ninguno de los programas insignias en el Plan Social Educativo parece atender de forma directa el tema de la capacidad crítica ni la formación de ciudadanos. Por ejemplo, los programas de alimentación escolar, educación inclusiva y dotación de uniformes y útiles escolares, siguen la línea de una política subsidiaria cuyo objetivo es atraer estudiantes y mantenerlos dentro del sistema educativo. El programa de alfabetización por su lado, tiene como propósito reducir el analfabetismo como condición para insertarse en el desarrollo social, económico y político. Los programas "Educación inicial", "Dignificación del Magisterio" y "Desarrollo Profesional" buscan mejorar las condiciones salariales, laborales y profesionales de los docentes. Por último, el programa "Infraestructura escolar" tiene como fin la reparación y dotación de equipo a los centros escolares.

Si bien es cierto que todos estos programas se caracterizan por la buena intención de mejorar las condiciones de la educación en El Salvador, también es cierto que ninguno de ellos podría traducirse de manera directa en una educación capaz de formar ciudadanos críticos y activos. Haría falta un programa insignia que haga explícito los procedimientos mediante los cuales se garantice el derecho a la educación y en particular, el derecho a una educación transformadora, acorde con lo que la Constitución Política de El Salvador demanda.

A pesar de todas las acciones que ambos planes educativos han impulsado en la última década, "El Salvador no ha conseguido construir una escuela pública de calidad que proporcione las bases para que las personas sean agentes de su propio desarrollo" (PNUD, 2013a, p. 138). Se identifican al menos tres obstáculos para lograr una educación transformadora: la falta de una visión de país, la poca inversión en la educación de los salvadoreños y una educación que no responde a los fines de la educación salvadoreña.

\section{OBSTÁCULOS PARA UNA EDUCACIÓN TRANSFORMADORA}

Conviene reformular en una pregunta la declaración con la cual se inició este artículo: ¿por qué El Salvador no ha conseguido brindar una educación capaz de proporcionar las bases para que las personas se vuelvan agentes de transformación social y den respuestas a la situación de violencia e inseguridad que vive el país? Aquí se identifican tres obstáculos:

a. El primero es la ausencia de una política educativa que corresponda a una visión de país. Parece que el interés de cada gobierno por crear sus propios programas de educación no ha permitido alcanzar acuerdos que construyan una visión a largo plazo en cuanto a educación. Algo de esto ya ha sido apuntado por el PNUD al señalar que, dado que las políticas educativas cambian con cada gobierno, "esto evita que se vean los frutos de los diferentes planes cuyos fines se traslapan y sus resultados se vuelven invisibles" (2013a, p. 143). Entonces, a menos que haya acuerdos sobre el ciudadano que se pretende formar para el tipo de sociedad deseada, así como un compromiso para impulsar planes y políticas educativas de largo plazo, cada gobierno seguirá implementando sus propias marcas y respondiendo más a intereses sectoriales, ideológicos o partidarios que a los fines de la educación de El Salvador.

b. El segundo obstáculo tiene que ver con la escasa inversión en la educación de los salvadoreños. En Latinoamérica, El Salvador es de los países que históricamente se han caracterizado por invertir muy poco en la educación de su pueblo. El diagnóstico del PNUD (2013b) señala que, entre 1906 y 1950, la inversión en educación y en salud (ambos rubros unidos) apenas fue del $13.6 \%$, pero otras instancias como seguridad y defensa gozaban del $23.8 \%$. Durante los años 1950 y 1979, la inversión en educación y salud subió al $35.6 \%$, pero la posterior guerra civil durante la década de los ochentas, nuevamente bajó, esta vez al 26.9\%, mientras que el ejército armado recibía el $23.6 \%$ del gasto público. No fue sino hasta el período de 1990 al 2012 
que, tanto educación como salud, tuvieron una inversión total del 32\%. Aunque ello representó una recuperación de la inversión en la educación, ese monto todavía era menor que la asignación hecha más de dos décadas atrás, y mucho menor de la que se necesitaría para mejorar el nivel de educación de los salvadoreños.

Evidentemente, la tendencia de la inversión en educación en los últimos años no ha sido al incremento. Las estadísticas muestran que solo en el 2009, tal porcentaje subió a 3.7\%, pero luego ha disminuido. Mientras otros países como Costa Rica y Cuba han invertido en educación arriba del $6 \%$ y del $13 \%$ del PIB respectivamente (Banco Mundial, 2014; UNESCO, 2013), en El Salvador hay una reducción: 3.5\% en 2010, 3.4\% en $2011,3.0 \%$ en 2012 y 3.4\% en 2013 (ERCA, 2014; Ministerio de Hacienda, 2012; Transparencia Fiscal, s.f.). Además, ERCA (2014) afirma que lo invertido en educación por cada salvadoreño ronda los \$100 en promedio desde el 2004 hasta el 2012. Esa cifra es claramente baja en la región centroamericana comparada con Costa Rica, que ha invertido $\$ 422$ en promedio por persona del 2004 al 2012 y que para este último año dedicó casi $\$ 700$ por persona (el más alto en Centroamérica). Estos números demuestran que el dinero destinado para la educación de los salvadoreños está muy por debajo del promedio en la región (5.2\%) y muy alejado de lo recomendado por la UNESCO, que ronda el $7 \%$ y $8 \%$ del PIB (PNUD, 2013b).

Sin duda, el gobierno salvadoreño podría aumentar esas cifras y mejorar así, la educación. Por ejemplo, en lugar de asignar fondos públicos a un Ministerio de la Defensa Nacional, que no representa una contribución para mejorar los problemas sociales del país (\$148.4 millones equivalente al $0.6 \%$ del PIB en el 2015), podría utilizarlos para atender los males crónicos en el sistema educativo, tales como analfabetismo, acceso limitado, deserción escolar y falta de preparación docente.

c. El tercer obstáculo para alcanzar una educación de calidad deriva de los planes educativos gubernamentales, que han estado orientados por fina- lidades distintas a las planteadas en los artículos 55 y 56 de la Constitución Política de El Salvador. Al revisar los dos programas de educación, se puede identificar que el primero se fundamenta en una idea de educación para la competitividad, mientras el segundo concibe la calidad de la educación a partir de la formación técnica y tecnología. En el primer caso -educación para la competitividad- su objetivo era lograr una educación no solo para ocupar puestos elevados en el ranking del índice de desarrollo humano, sino también para alcanzar un alto y sostenido crecimiento económico. Si bien las líneas estratégicas en este plan se enfocaron en el acceso, la competitividad, la productividad, la eficiencia y la efectividad, ninguna abordaba de forma particular la formación de ciudadanos críticos y activos que promuevan valores humanos tales como el respeto a la vida, la tolerancia y la convivencia.

En cuanto al segundo plan de educación, aunque en el discurso se menciona el deseo de ofrecer una educación transformadora, que forme ciudadanos con juicio crítico, no se declara cómo se lograrán a partir de sus líneas estratégicas. Como se mencionó antes, en este plan educativo la calidad está en función de la dotación de computadoras portátiles a estudiantes y docentes. Sin embargo, como se apuntó antes, mejorar la educación no se puede garantizar por este tipo de acciones cuando aún existen carencias de infraestructura apropiada para ello y, sobre todo, falta de capacidad pedagógica para un uso pertinente de la tecnología. Sin la preparación docente apropiada y sin la formación didáctico-metodológica y claridad pedagógica, la tecnología no ayuda a responder a los problemas de convivencia que actualmente enfrenta la sociedad salvadoreña. Mejorar la educación requiere invertir no solo en infraestructura y tecnología, sino también en la profesionalización de los educadores, pues la enseñanza y el aprendizaje están en función directa de quienes la llevan a cabo en las aulas.

El Salvador no logrará que sus ciudadanos sean capaces de realizar cambios en las condiciones y problemas sociales actuales a partir de acciones y programas orientados únicamente por la competitividad o la actualización tecnológica. No basta con pretender que los estudiantes se constituyan en mano 
de obra calificada, cuya utilidad sea estar al servicio del mercado. Tampoco es suficiente con el simple hecho de dar computadoras a todos los estudiantes y profesores. Estas acciones, aunque necesarias, son insuficientes para una educación orientada a la formación de ciudadanos críticos y activos e incapaces, por sí solas, de alcanzar los fines de la educación planteados en la Constitución Política de El Salvador.

\section{CONCLUSIÓN}

La Constitución Política de El Salvador, en su artículo 55, establece que la finalidad de la educación es crear las condiciones para que esta sociedad pueda vivir en paz y en armonía. El propósito declarado demanda el desarrollo integral de la persona y la construcción de una sociedad participativa, justa y humana a partir de la promoción de los derechos humanos y el combate a la violencia, el odio y la intolerancia. Además, el artículo 56 obliga a formar ciudadanos de beneficio para la sociedad salvadoreña. Sin embargo, para ambos casos, la realidad educativa demuestra que la educación salvadoreña permanece alejada de estas declaraciones constitucionales.

A pesar de que dicha finalidad se ha retomado en los discursos que emanan de los diversos agentes e instituciones educativas, no se ha logrado pasar del ámbito simbólico a la intervención concreta. Ejemplo de esto es la grave situación de violencia e inseguridad que actualmente imperan en la sociedad salvadoreña, y que avanzan aceleradamente sin que la educación haya podido contribuir de forma certera ni dar una respuesta pertinente ante dicho problema. Y aunque es cierto que no se podría esperar que la educación resuelva todos los problemas de la sociedad salvadoreña, también es cierto que garantizar el derecho a una educación transformadora puede contribuir con la solución ante situaciones de violencia, intolerancia y desigualdad social. No obstante, tal derecho se ve obstaculizado tanto por un bajo acceso al sistema educativo, alta deserción escolar, analfabetismo de buena parte de la población y limitada preparación docente, como por la baja inversión en la educación y la falta de una visión de país que apueste de forma decidida por invertir en la formación de sus ciudadanos. Esto ha ocasionado que los planes educativos gubernamentales sean orientados por propósitos diferentes a lo establecido por la Constitución Política, es decir, hacia la competitividad laboral o la actualización tecnológica, por delante de la formación de ciudadanos capaces de transformar su entorno y dar respuestas a los problemas sociales.

Sentar las bases para que los salvadoreños se conviertan en ciudadanos capaces de cambiar su entorno social requiere revalorar la finalidad de la educación salvadoreña y garantizar una educación transformadora. Por una parte, el ciudadano útil que la Constitución Política de El Salvador demanda, debe ser crítico y activo. Es decir, con capacidad de cuestionar el orden social existente para orientar sus decisiones, actuar en favor de la convivencia y la diversidad y rechazar la violencia y exclusión, presentes en la sociedad salvadoreña actual.

Por otra parte, lograr ese ciudadano no será posible a menos que las políticas educativas estén orientadas a garantizar una educación transformadora. Dicha acción educativa transformadora tiene como idea subyacente que para la formación de ciudadanos críticos y activos es imprescindible que los estudiantes tengan oportunidades para desarrollar el pensamiento crítico, el cual, valga anotar, resulta un componente indispensable para una educación que tenga como finalidad la transformación de la sociedad mediante la experiencia significativa y el sentido de responsabilidad.

Si se desea formar un ciudadano crítico ha de ejercitarse el análisis social estructural, el razonamiento independiente, el desarrollo de conciencia crítica y el examen de las relaciones, el comportamiento de los individuos y de las estructuras que configuran la injusta desigualdad social y exclusión que dan origen a muchos de los problemas sociales que la población salvadoreña actualmente enfrenta, como la violencia e inseguridad.

Por último, la formación de un ciudadano activo parte de la comprensión y del reconocimiento del derecho que cada estudiante tiene para expresarse en experiencias participativas de aprendizaje caracterizadas por el respeto y el trato horizontal. Lo anterior es condición para que, mediante la reflexión y el análisis crítico, los estudiantes orienten la toma de decisión y la actuación que favorezca la convivencia, 
la aceptación de la diversidad cultural, el respeto al medioambiente así como el rechazo a las diferentes formas de violencia y exclusión que ellos enfrentan.

\section{REFERENCIAS BIBLIOGRÁFICAS}

Asamblea Legislativa de El Salvador. (s.f.). Ley General de educación. Obtenido de http://www.asamblea.gob.sv/eparlamento/indice-legislativo/buscador-de-documentos-legislativos/ ley-general-de-educacion

AUPRIDES. (s.f.). ¿Quiénes somos? Obtenido el 14 de junio de 2015, de http://www.auprides.org.sv/index. php?option=com_content $\&$ view $=$ article $\& i d=25 \&$ Item id $=27$

Banco Mundial. (2015). Datos: Gasto público en educación, total (\% del PIB). Recuperado el 20 de mayo de 2015, de http://datos.bancomundial.org/: http://datos.bancomundial. org/indicador/SE.XPD.TOTL.GD.ZS/countries/CR-SV-GT$\mathrm{HN}-\mathrm{NI}-\mathrm{CU}$ ?display=graph

BBC Mundo. (29 de Diciembre de 2014). Los 3.800 asesinatos que tienen en alerta a El Salvador. Recuperado el 20 de Mayo de 2015, de http://www.bbc.co.uk/: http://www. bbc.co.uk/mundo/noticias/2014/12/141222_america_latina_salvador_mara_pandillas_18_amv

Bean, J. C. (2011). Engaging ideas. San Francisco: Jossy-Bass.

Bevan, R. (2009). Expanding rationality: the relation between epistemic virtue and critical thinking. Educational Theory, 29(2), pp. 167-179.

Boghossian, P. (2012). Critical thinking and constructivism: mambo dog fish to the banana patch. Journal of Philosophy of Education, 46 (1), pp. 73-84.

Corte Suprema de Justicia de El Salvador. (16 de Diciembre de 1983). Constitución de la República de El Salvador. Diario Oficial N²34, tomo N²81.

Crenshaw, P., Halke, E. y Harper, S. L. (2011). Producing intellectual labor in the classroom: the utilization of a critical thinking model to help students take command of their thinking. Journal of College Teaching \& Learning, 8(7), pp. 13-26.

Delgado, P. (2010). Educación, democracia y ciudadanía en el siglo XXI desde el proyecto político educativo de Paulo Freire. Revista Fuentes, pp. 140-153.

Doughty, H. A. (2007). The limits of critical thinking. The Innovation Journal: The Public Sector Innovation Journal. 11(3), pp. 1-10.

Dubet, F. (2005). La escuela de las oportunidades ¿Qué es una escuela justa? Barcelona: Editorial Gedisa, S.A.
Dwyer, C. P., Hogan, M. J. y Stewart, I. (2012). An evaluation of argument mapping as a method of enhancing critical thinking performance in e-learning environments. Metacognition \& Learning, 7(3), 219-244.

El Salvador cerró el 2014 con casi 4,000 homicidios. (2015, 2 de enero). El Diario de Hoy. Recuperado el 20 de mayo de 2015, de http://www.elsalvador.com/: http:// www.elsalvador.com/mwedh/nota/nota_completa. asp?idCat=47859\&idArt=9360619

2015 supera cifras anuales de homicidos registradas en los últimos seis años. (2015, 9 de septiembre). El Diario de Hoy. Recuperado el 9 de Septiembre de 2015, de http://www.elsalvador.com/articulo/sucesos/2015-supera-cifras-anualeshomicidos-registradas-los-ultimos-seis-anos-86937 \#.VfDHjnY7A3s

Elder, L. y Paul, R. (2013). Critical thinking: intellectual standards essential to reasoning well within every domain of thought. Journal of Developmental Education, 36(3), pp. 34-35.

ERCA. (2014). Estadísticas de Centroamérica 2014. San José Costa Rica: Programa Estado de la Nación - Región.

Facione, P. (2014). insightassessment.com. Obtenido de http:// www.insightassessment.com/CT-Resources/Teaching-Forand-About-Critical-Thinking/Critical-Thinking-What-It-Isand-Why-It-Counts/Critical-Thinking-What-It-Is-and-WhyIt-Counts-PDF

Facione, P. A. (1990). Critical thinking: a statement of expert consensus for purposes of educational assessment and instruction. Millbrae: The California Academic Press.

Ferraz, M. (2012). De la pedagogía oficial a la pedagogía crítica. Revista Iberoamericana de Educación. 58(4), pp. 2-16.

Giroux, H. (1990). Los profesores como intelectuales: hacia una pedagopgía crítica del aprendizaje. Barcelona: PAIDÓs.

Giroux, H., \& McLaren, P. (1998). Educación de maestros y la política del compromiso: el caso pro-escolarización democrática. En H. Giroux, \& P. McLaren, Sociedad, cultura y educación (págs. 87-135). Buenos Aires: Miño y Dávila Editores.

Gutiérrez, F. (1985). Educación como praxis política. Siglo Veintiuno editores.

Haydon, G. (2003). Enseñar valores: un nuevo enfoque. Madrid: Ediciones Morata S. L.

Johnson, L., \& Morris, P. (2010). Towards a framework for critical citizenship education. The Curriculum Journal, 21(1), pp. 77-96. 
Una educación transformadora para El Salvador: propuesta y obstáculos para alcanzarla

Levine, T. H. (2010). Socializing future social studies teachers and K-12 students: whether, when, and why. The Social Studies, 10, pp. 69-74.

Lim, L. (2011). Beyond logic and argument analysis: critical thinking, everyday problems and democratic deliberation in Cambridge International Examinations' Thinking Skills curriculum. Journal of Curriculum Studies, 43(6), pp. 783-807.

MacLaren, P. y Giroux, H. (2003a). La formación de los maestros en una esfera contra-pública: notas hacia una redefinición. En P. MacLaren, Pedagogía, identidad y poder (págs. 11-48). Santa Fe: HomoSapiens Ediciones.

MacLaren, P. y Giroux, H. (2003b). Desde los márgenes: geografías de la identidad, la pedagogía y el poder. En P. MacLaren, Pedagogía, identidad y poder. (págs. 49-85). Santa Fe: HomoSapiens Ediciones.

McCowan, T. (2012). Opening spaces for citizenship in higher education: three initiatives in English universities. Studies in Higher Education, 37(1), pp. 51-67.

Más de 80 homicidios en inicio de Semana Santa en El Salvador. (1 de Abril de 2015). La Prensa Gráfica. Recuperado el 20 de mayo de 2015, de http://www.laprensagrafica. com/: http://www.laprensagrafica.com/2015/04/01/mas-de80-homicidios-en-inicio-de-semana-santa-en-el-salvador

MINED. (2005). Plan Nacional de Educación 2021: Fundamentos. San Salvador.

MINED. (2008). Currículo al servicio del aprendizaje: aprendizaje por competencias. 2a Edición. San Salvador, El Salvador: Ministerio de Educación.

MINED. (2009). https://www.mined.gob.sv. Obtenido el 15 de junio de 2015, de http://www.mined.gob.sv/index.php/institucion/marco/historia.html

MINED. (2009). Transformación de la educación: Programa Social Educativo 2009-2014 "Vamos a la Escuela". San Salvador: MINED.

Ministerio de Hacienda de la República de El Salvador. (2012). Presupuesto Estimado 2013. Recuperado el 20 de mayo de 2015, del sitio http://www.mh.gob.sv/: http://www.mh.gob. sv/portal/page/portal/PMH/Institucion/Ministro_de_Hacienda/Presentaciones/Presentacion_Presupuesto_2013_Vdefinitiva.pdf

Nussbaum, M. C. (2006). Education and democratic citizenship: capabilitiesand quality education. Journal of Human Development, 7(3), pp. 385-395.

Olivares, S., Saiz, C. y Rivas, S. F. (2013). Encouragement for thinking critically. Electronic Journal of Research in Educational Psychology, 11(2), pp. 367-393.
Perafán, B. (2014). Formación ciudadana en la universidad: una propuesta para la acción. Revista de Derecho Público, 32., pp. 2-22.

PNUD. (2013a). Informe sobre el desarrollo humano El Salvador 2013: Imaginar un nuevo país. Hacerlo posible. Diagnóstico y propuesta. San Salvador: Programa de las Naciones Unidas para el Desarrollo (PNUD).

PNUD. (2013b). Informe sobre Desarrollo Humano en El Salvador 2013 (resumen). San Salvador: Programa de las Naciones Unidas para el Desarrollo (PNUD).

Posada, L. (2013). Pensamiento crítico y ruptura cognitiva en la educación. Escritos en la Facultad, pp. 34-35.

Rodríguez, C. R. (2006). La vigencia de la educación como mecanismo de movilidad social en la sociedad del conocimiento. Revista Regional de Investigación Educativa (3), Otoño, pp. 66-80.

Sacristán, J., y Pérez, A. (2003). Comprender y transformar la enseñanza. Madrid: Morata.

Sistema de la Integración Centroamericana. (27 de junio de 2014). Política Educativa Centroamericana 2013-21. Punta Cana, República Dominicana: CECC/SICA.

Steur, J., Jansen, E. y Hofman, W. (2012). Graduateness: an empirical examination of the formative function of university education. High Educ, 64, pp. 861-874.

Tenti, E. (2009). Diversidad cultural y ciudadanía activa: consideraciones sociológicas. En E. Tenti Fanfani, Desigualdad cultural, desigualdad social y estatregias de políticas educativas. (págs. 79-110). Buenos Aires: IIPE.UNESCO.

Transparencia Fiscal. (s.f.). http://www.transparenciafiscal.gob. sv/. Obtenido de http://www.transparenciafiscal.gob.sv/ptf/ es/PresupuestosPublicos/GuasdelPresupuestoparaelciudadano.html

UNESCO. (2013). Situación educativa de América Latina y el Caribe: hacia la educación de calidad para todos al 2015. Santiago: OREALC/UNESCO.

Universidad de El Salvador. (s.f.). Nuestra Universidad: Visión y Misión. Obtenido el 14 de mayo de 2015, de http://www. ues.edu.sv/nuestra-universidad

Universidad Don Bosco. (s.f.). Institucional: Modelo Educativo. Obtenido de http://www.udb.edu.sv/udb/index.php/pagina/ ver/modelo_educativos

\section{NOTA}

i La autoregulación se entiende como el autoexamen y la autocorrección (Facione, 1990). 